\title{
Combined Interlaminar and Paraisthmic Approach for Co-existing Intracanal and Foraminal Lesion
}

\author{
Jung-Sup Lee', Jong-Yun Woo ${ }^{2}$, Jee-Soo Jang', II-Tae Jang ${ }^{2}$ \\ ${ }^{I}$ Department of Neurosurgery, Suwon Nanoori Hospital, Suwon-si, Gyeonggi-do, \\ ${ }^{2}$ Department of Neurosurgery, Seoul Nanoori Hospital, Seoul, Korea
}

\begin{abstract}
Objective: Stenosis or herniated nucleus pulposus (HNP) occupying lumbar intracanal and foraminal area is an important cause of double radicular symptoms. Using the combined interlaminar and paraisthmic approach, we performed decompression surgery in patients with co-existing intracanal and foraminal lesions. The objective of this study is to describe usefulness and outcome of combined interlaminar and paraisthmic approach surgery and to analysis the cause of poor outcome. Methods: Between Apr 2009 and Apr 2014, 78 patients (42 males and 36 females) with intracanal and foraminal lesions were enrolled in this study. Patients with a vacuum disc, spondylolisthesis, instability or an isthmic defect on the preoperative dynamic view radiograph were excluded from this study. All patients underwent surgery through a combined approach for discectomy and decompression. The outcome of surgery was evaluated and classified into excellent, good, fair and poor. Results: The results were excellent in 53 patients, good in 9, fair in 6 and poor in 10 during the follow-up. The outcome of the combined approach was excellent to fair in $87 \%$ (68 of 78) patients in our study. In the poor outcome group, three patients complained of early-onset relapsed pain ( $<1$ month) and another seven patients complained of delayed-onset pain (>3 months).

Conclusion: Combined approach for both intracanal and foraminal area lesions may be useful if selectively performed on patients whose facet joint is relatively intact, and that it is worthy of consideration as an alternative to fusion surgery; however, further studies are needed.
\end{abstract}

Key Words: Spinal stenosis · Radiculopathy $\cdot$ Lumbar

\section{INTRODUCTION}

The lumbar spine can be divided anatomically into the central and lateral areas. Lesions in the central area (intracanal lesions) have traditionally been managed through a standard interlaminar approach. Lesions in the lateral area (foraminal lesions) occasionally involve the exiting (upper) nerve root within the neuroforamen and exposed through an extraspinal intertransverse approach ${ }^{6,15,16,18)}$. Spinal stenosis or herniated nucleus pulposus $(\mathrm{HNP})$ extending into both the intracanal

\footnotetext{
- Received: November 15, 2015 • Revised: November 29, 2015

- Accepted: November 30, 2015

Corresponding Author: Jung-Sup Lee, MD, PhD

Department of Neurosurgery, Suwon Nanoori Hospital, 295,

Jungbu-daero, Yeongtong-gu, Suwon-si, Gyeonggi-do 16503, Korea

Tel: +82-31-1688-9797, Fax: +82-31-8065-9701

E-mail: lis31133@hanmail.net

$\otimes$ This is an Open Access article distributed under the terms of the Creative

Commons Attribution Non-Commercial License (http://creativecommons.org/

licenses/by-nc/3.0/) which permits unrestricted non-commercial use, distribution,

and reproduction in any medium, provided the original work is properly cited.
}

and foraminal areas causes the development of biradicular symptoms, and several authors managed such conditions by performing a medial facetectomy or a total facetectomy ${ }^{4,7}$. Medial facetectomy has the drawback of controlling the neuroforamen on one side alone; thus, the presence of disc remnants within or outside the foramen cannot be excluded ${ }^{17}$. Additional partial facet removal in a lateral direction can lead to instability, and sometimes fusion surgery is required ${ }^{13)}$. Therefore, several surgeons perform total facetectomy with fusion surgery for lesions existing in both the intracanal and foraminal areas as the proper treatment ${ }^{4,12,16}$. We performed the combined interlaminar and paraisthmic approach as an alternative to fusion surgery. This sporadically reported approach has the advantage of allowing the foramen to be inspected on both sides, and of being a more time- and cost-saving procedure than total facetectomy with fusion surgery. In this study, we report our experience with a combined interlaminar and paraisthmic approach for lesions existing in both the intracanal and foraminal areas. The objective of this study was to describe the usefulness and outcome of the combined interlaminar and paraisthmic approach and to analyze the cause of poor outcome. 


\section{MATERIALS AND METHODS}

Between April 2009 and April 2014, 78 patients (42 males and 36 females) with intracanal and foraminal stenosis or herniated nucleus pulposus (HNP) were enrolled in this study (Table 1). All patients had unilateral lesions and were refractory to conservative treatment. Patients with a vacuum disc, spondylolisthesis, instability, or an isthmic defect on the preoperative dynamic view radiograph were excluded from this study. All patients underwent surgery through a combined interlaminar and paraisthmic approach for discectomy and decompression. The average age of the patients was 67 years (range 33-82 years). All data were collected through a retrospective review of the charts and radiologic images. Advanced imaging evaluations such as computed tomography (CT) and magnetic resonance imaging (MRI) were taken for the preoperative diagnosis of the intracanal and foraminal lesions. The CT images were reconstructed into parasagittal images of the foramen, and allowed the detection of bony encroachment into the foramen. The parasagittal and axial magnetic resonance images were evaluated for the presence of intracanal and foraminal lesions (Fig. 1). A reduced size of the foramen

Table 1. Demographic features of the 78 patients who underwent combined-approach surgery

\begin{tabular}{ccc}
\hline \hline Characteristics & No. of patients $(\mathrm{n}=78)$ & $\%$ \\
\hline Sex & 42 & 54 \\
Male & 36 & 46 \\
Female & & \\
Age, years & 4 & 5 \\
$30-40$ & 5 & 6 \\
$41-50$ & 19 & 25 \\
$51-60$ & 24 & 31 \\
$61-70$ & 21 & 27 \\
$71-80$ & 5 & 6 \\
$81-$ & &
\end{tabular}

Table 2. Modified Odom's criteria

\begin{tabular}{cl}
\hline Grade & \multicolumn{1}{c}{ Definition } \\
\hline Excellent & $\begin{array}{l}\text { All preoperative symptoms are relieved; the patient is } \\
\text { able to perform daily occupational tasks and activities } \\
\text { without impairment. }\end{array}$ \\
Good & $\begin{array}{l}\text { Minimal persistence of preoperative symptoms; the } \\
\text { patient is able to perform daily occupational tasks and } \\
\text { activities without significant interference. }\end{array}$ \\
Fair & $\begin{array}{l}\text { Relief of some preoperative symptoms; however, daily } \\
\text { occupational tasks and activities remain significantly } \\
\text { limited. }\end{array}$ \\
Poor & Symptoms and signs are unchanged or become worse.
\end{tabular}

and obliteration of epidural fat surrounding the exiting nerve root were considered as evidence of significant foraminal stenosis. Postoperative serial dynamic view radiographs were taken to evaluate the development of instability or isthmic defect. The clinical outcome of surgery was evaluated and classified into excellent, good, fair, and poor, on the basis of modified Odom's criteria (Table 2).

\section{Surgical Technique}

The first step in the operation is the standard interlaminar approach to the intracanal lesions. A C-arm fluoroscope is used to verify the correct position of the incision. After the incision, the lumbodorsal fascia is detached bluntly from the attachment to the spinous process. Erector spinae muscles at the involved level are dissected subperiosteally from the spinous process and lamina. Those muscles are retracted laterally over the facet joint carefully. After the application of a Caspar microlumbar discectomy retractor, partial laminectomy and decompression of the intracanal area are performed. In the second part of the operation, the paravertebral muscle is retracted further laterally to visualize the lateral border of the isthmus and the upper portion of the facet joint. With a high-speed drill, the uppermost part of the facet joint and the lateral margin of the isthmus are drilled. The amount of facet joint resection should not exceed 50\%. Then, the intertransverse and foraminal ligaments are excised to expose the lesion lateral to the foramen. The affected nerve root is then followed along the neuroforamen. Sufficient nerve root decompression must be carefully confirmed by using a foraminal probe. With this combined approach, it is possible to maintain the integrity of the facet joint and obtain sufficient exploration and decompression of the foramen (Fig. 2).
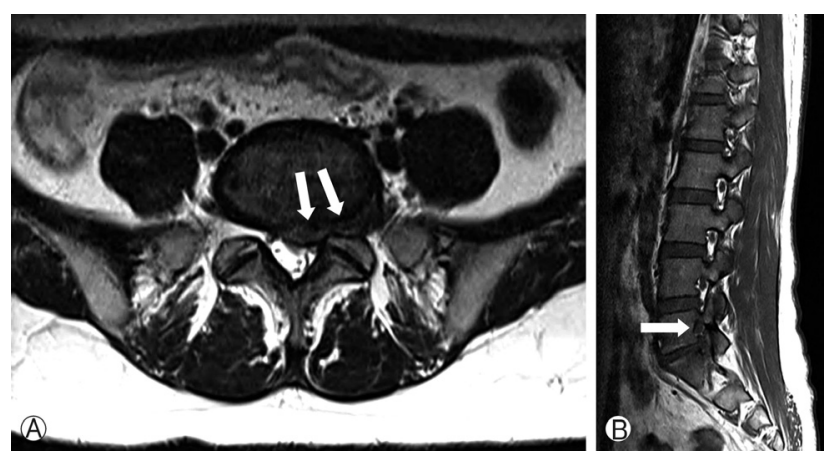

Fig. 1. Preoperative magnetic resonance (MR) images obtained at level L5-S1. (A) Axial T2-weighted MR image shows a herniated disc effacing both the central and the left foraminal zones. (B) Parasagittal T1-weighted image showing a left L5-S1 foraminal stenosis with perineural fat obliteration surrounding the nerve root compared with adjacent levels. 


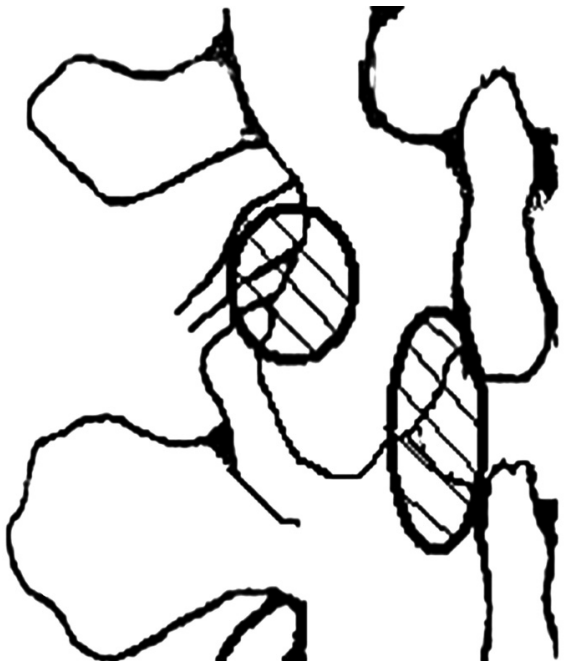

Fig. 2. Anatomical diagram showing the bony resection area (circle) of the combined interlaminar and paraisthmic approach for decompression surgery.

Table 3. Level of operation

\begin{tabular}{ccc}
\hline \hline Level & No. of patients $(n=78)$ & $\%$ \\
\hline L2-3 & 2 & 3 \\
L3-4 & 10 & 13 \\
L4-5 & 51 & 65 \\
L5-S1 & 15 & 19 \\
\hline
\end{tabular}

Table 4. Outcome based on modified Odom's criteria

\begin{tabular}{lcc}
\hline \hline Grade & No. of patients $(\mathrm{n}=78)$ & $\%$ \\
\hline Excellent & 53 & 68 \\
Good & 9 & 12 \\
Fair & 6 & 7 \\
Poor & 10 & 13 \\
\hline
\end{tabular}

\section{RESULTS}

All 78 patients complained severe radicular leg pain, and 55 patients had combined back pain. The levels involved in the operation were L2-3 (2 patients), L3-4 (10 patients), L4-5 (51 patients), and L5-S1 (15 patients) (Table 3). The mean follow-up period was 13.4 months. The outcomes based on modified Odom's criteria were excellent in 53 patients, good in 9, fair in 6, and poor in 10 during the follow-up (Table 4). There were no surgery-related complications. Lumbar dynamic view radiography was performed in all patients at various times after the surgery, and instability was found in five patients and isthmus fracture was found in six patients. However, no patients required revision surgery for poor outcome. In the poor outcome group, three patients complained of early-onset relapsed pain ( $<1$ month), and the cause of pain was a technical error related to insufficient foraminal decompression. Another seven patients complained of delayed-onset pain (3 months after the operation), and the pain was due to recurrent herniated nucleus pulposus and adhesion. Patients with earlyonset pain underwent fusion surgery for a wider decompression, and four patients with delayed-onset relapsed pain underwent revision discectomy and adhesiolysis; however, the remaining three patients did not return to our hospital for revision surgery (Table 5). All seven patients who underwent revision surgery showed improvement at the last follow-up.

\section{DISCUSSION}

Lesions that extend to the intracanal and foraminal areas can cause double radicular compression ${ }^{17}$. Such lesions are difficult to treat with surgery. For lesions in the foraminal or the extraforaminal area, many authors perform decompre-

Table 5. Summary of patients with poor outcome after the combined-approach surgery

\begin{tabular}{ccccclcl}
\hline \hline Patients No. & Age & Sex & Level & Recurrence duration (mo) & Recurred site & Recurred character & Revision surgery \\
\hline 1 & 66 & F & $L 4-5$ & 5 & Foraminal & HNP & Decompression \\
2 & 64 & F & $L 4-5$ & $<1$ & Foraminal & I.D. & Fusion \\
3 & 49 & M & $L 5-6$ & 3 & F/u loss & F/u loss & F/u loss \\
4 & 76 & M & $L 4-5$ & 5 & Intracanal & Adhesion & Decompression \\
5 & 59 & $M$ & $L 4-5$ & $<1$ & Foraminal & I.D. & Fusion \\
6 & 65 & $M$ & $L 4-5$ & 6 & F/u loss & F/u loss & F/u loss \\
7 & 73 & F & $L 4-5$ & 8 & F/u loss & F/u loss & F/u loss \\
8 & 77 & F & $L 3-4$ & 3 & Intracanal & HNP & Decompression \\
9 & 42 & $M$ & $L 4-5$ & 6 & Intracanal & HNP & Decompression \\
10 & 62 & $M$ & $L 4-5$ & $<1$ & Foraminal & I.D. & Fusion \\
\hline
\end{tabular}

F/u, follow-up; HNP, herniated nucleus pulposus; I.D., insufficient decompression. 
ssion surgery with an extraspinal intertransverse approach; however, this approach only allows the exiting nerve root to be decompressed ${ }^{19,20)}$. Some authors continue to perform surgery with the anatomically familiar posterior midline interlaminar approach to decompress the intracanal and intervertebral foramen ${ }^{1,5,7}$. However, it is possible for unremoved disc fragments to remain within or outside the foramen, and if medial facetectomy is performed aggressively to have sufficient control over the foramen, it can cause instability ${ }^{4,9,12)}$. Consequently, these approaches share the disadvantage of controlling the neuroforamen only unilaterally ${ }^{17}$. Although total facetectomy is recommended as an appropriate approach for foraminal decompression, revision fusion surgery is sometimes required because instability can occur ${ }^{4,7,9,12)}$. Even if facet joint destruction does not cause acute instability, total facetectomy can modify the axial loading path in the three columns of the spine, and cause postoperative lower-back pain due to degeneration in the adjacent disco-ligamentous structures" ${ }^{97}$. For this reason, some authors believe that fusion surgery is necessary when a total facetectomy is performed ${ }^{4,12)}$. However, fusion surgery costs more, results in a higher amount of blood loss, and requires a longer surgery time and hospital stay than decompression surgery ${ }^{2,3}$. And it is well known that the fusion surgery may accelerate degenerative process in the adjacent disco-ligamentous structures ${ }^{14)}$.

A few reports have described the advantages of combinedapproach surgery ${ }^{10,11,14,17)}$. The combined-approach surgery makes it possible to completely control the neuroforamen and confirm the full extent of disc herniation in the surgical field of view ${ }^{11,14)}$. Additionally, this approach allows accessing the upper and lower nerve roots without destroying the facet joint or affecting spinal stability ${ }^{4,10,11,14,17)}$. In this combined-approach surgery, laminectomy is first performed with an interlaminar approach, and after the traversing nerve root is confirmed, the intracanal lesion is treated. In the process, the proximal portion of the exiting nerve root is confirmed during laminectomy, which can be helpful when the root is confirmed in the foramen ${ }^{17}$. Next, a paraisthmic approach is performed to access outside of foramen along the laminar, which provides the surgeon an anatomically familiar surgical field of view. When the lateral portion of the isthmus and the uppermost part of the facet joint are drilled, and the exposed intertransverse and foraminal ligaments are dissected, the exiting nerve root surrounded by adipose tissues can be confirmed, as well as the foraminal lesion. Even in patients with severe foraminal stenosis, movement of the proximal portion of the exiting nerve root is communicated to the extraforaminal portion of the nerve. Thus, when the surgeon moves the proximal portion of the exiting nerve root while observing the extraforaminal area, the surgeon can accurately ascertain the relation between the lesion and the $\operatorname{root}^{17}$.

In our study with a combined interlaminar and paraisthmic approach, the outcomes of the surgery were excellent to fair in $87 \%$ of the patients. The advantages of this surgical approach are that the facet joint is saved and instability is prevented. However, in 11 patients, we confirmed isthmus fracture or instability on postoperative dynamic view radiographs, and we think that this may be because the wide resection performed to achieve sufficient decompression could have weakened the facet joint or the isthmus. Most patients were asymptomatic, and the problem did not require revision surgery in any of these cases. In 10 patients with poor outcome, the age, sex, or surgery level was not statistically significant. But follow-up CT or MRI imaging showed a technical error such as insufficient foraminal decompression in three patients with earlyonset relapsed pain ( $<1$ month). Of seven patients with delayedonset pain, four had recurrent HNP or adhesion, and three were lost to follow-up. Insufficient decompression is the most frequently occurring error in spinal decompression surgery ${ }^{8)}$. Thus, to improve surgical outcomes, an effort should be made to reduce technical errors. This study has a short-term followup ( $~ 1$ year). Although isthmus fracture or instability does not manifest in the first year, degenerative changes may occur with time and cause pain in the future. Therefore, for an accurate prognosis, a larger number of patients and a longer followup would be needed. Overall, we think that surgery with this approach may be useful if selectively performed on patients whose facet joint is relatively intact, and that it is worthy of consideration as an alternative to fusion surgery; however, further studies are needed.

\section{CONCLUSION}

The outcome of the combined interlaminar and paraisthmic approach was excellent to fair in $87 \%$ (68 of 78 ) patients in our study. It is a useful management method for select patients who have lesions that occur in both the intracanal and foraminal areas, and we think that it is a worthy alternative to total facetectomy with fusion surgery. The procedure allows sufficient foramen exploration with a minimally invasive approach, and preserves the integrity of the facet joint. We believe that improved surgical outcome can be attained if efforts are made to achieve sufficient foraminal decompression. A longer follow-up on a large series of patients is needed.

\section{REFERENCES}

1. Abdullah AF, Ditto EW 3rd, Byrd EB, Williams R: Extreme- 
lateral lumbar disc herniations. Clinical syndrome and special problems of diagnosis. J Neurosurg 41:229-234, 1974

2. Carreon LY, Puno RM, Dimar JR 2nd, Glassman SD, Johnson JR: Perioperative complications of posterior lumbar decompression and arthrodesis in older adults. J Bone Joint Surg Am 85-A:2089-2092, 2003

3. Cho KJ, Suk SI, Park SR, Kim JH, Kim SS, Lee TJ, et al: Short fusion versus long fusion for degenerative lumbar scoliosis. Eur Spine J 17:650-656, 2008

4. Epstein NE: Evaluation of varied surgical approaches used in the management of 170 far-lateral lumbar disc herniations: indications and results. J Neurosurg 83:648-656, 1995

5. Epstein NE: Foraminal and far lateral lumbar disc herniations: surgical alternatives and outcome measures. Spinal Cord 40:491500, 2002

6. Faust SE, Ducker TB, VanHassent JA: Lateral lumbar disc herniations. J Spinal Disord 5:97-103, 1992

7. Garrido E, Connaughton PN: Unilateral facetectomy approach for lateral lumbar disc herniation. J Neurosurg 74:754-756, 1991

8. Getty CJ: Lumbar spinal stenosis: The clinical spectrum and the results of operation. J Bone Joint Surg Br 62-B:481-485, 1980

9. Haher TR, O'Brien M, Dryer JW, Nucci R, Zipnick R, Leone DJ: The role of the lumbar facet joints in spinal stability. Identification of alternative paths of loading. Spine (Phila Pa 1976) 19:2667-2670, 1994

10. Hassler W, Brandner S, Slansky I: Microsurgical management of lateral lumbar disc herniations: combined lateral and interlaminar approach. Acta Neurochir (Wien) 138:907-910, 1996

11. Hejazi N, Witzmann A, Hergan K, Hassler W: Combined transarticular lateral and medial approach with partial facetectomy for lumbar foraminal stenosis. Technical note. J Neurosurg 96: 118-121, 2002

12. Kunogi J, Hasue M: Diagnosis and operative treatment of intraforaminal and extraforaminal nerve root compression. Spine (Phila Pa 1976) 16:1312-1320, 1991

13. Bae KJ, Choi ES, Jung ES, Son BG, Shin JH, Kim MJ, et al: Analysis of surgical outcome of lumbar stenosis over 2 year follow-up. Korean J Spine 3:75-81, 2006

14. Kwon YJ: Minimally invasive combined interlaminar and paraisthmic approach for symptomatic lumbar foraminal stenosis: Surgical technique and preliminary results. J Korean Neurosurg Soc 42:11-15, 2007

15. Monteiro A, Lefevre R, Pieters G, Wilmet E: Lateral decompression of a pathological disc in the treatment of lumbar pain and sciatica. Clin Orthop Relat Res 238:56-63, 1989

16. O'Brien MF, Peterson D, Crockard HA: A posterolateral microsurgical approach to extreme-lateral lumbar disc herniation. J Neurosurg 83:636-640, 1995

17. Paolini S, Ciappetta P, Raco A, Missori P, Delfini R: Combined intra-extracanal approach to lumbosacral disc herniations with bi-radicular involvement. Technical considerations from a surgical series of 15 cases. Eur Spine J 15:554-558, 2006

18. Siebner HR, Faulhauer K: Frequency and specific surgical management of far lateral lumbar disc herniations. Acta Neurochir (Wien) 105:124-131, 1990

19. Tessitore E, de Tribolet N: Far-lateral lumbar disc herniation: The microsurgical transmuscular approach. Neurosurgery 54: 939-942, 2004

20. Wiltse LL: The paraspinal sacrospinalis-splitting approach to the lumbar spine. Clin Orthop Relat Res 91:48-57, 1973 